\title{
Synchronization in functional networks of the human
}

\section{brain}

3 Philipp Hövel* • Aline Viol · Philipp

4 Loske • Leon Merfort • Vesna Vuksanović*

6 Received: date / Accepted: date

7 Abstract Understanding the relationship between structural and functional or-

8 ganization represents one of the most important challenges in neuroscience. An

9 increasing amount of studies show that this organization can be better under-

10 stood by considering the brain as an interactive complex network. This approach

11 has inspired a large number of computational models that combine experimental

Philipp Hövel

Institute of Theoretical Physics, Technische Universität Berlin, Hardenbergstraße 36, 10623 Berlin, Germany

Bernstein Center for Computational Neuroscience Berlin, Humboldt-Universität zu Berlin, Philippstraße 13, 10115 Berlin, Germany

E-mail: phoevel@physik.tu-berlin.de

Aline Viol

Institute of Theoretical Physics, Technische Universität Berlin, Hardenbergstraße 36, 10623 Berlin, Germany

Bernstein Center for Computational Neuroscience Berlin, Humboldt-Universität zu Berlin, Philippstraße 13, 10115 Berlin, Germany

E-mail: aline.viol@bccn-berlin.de

Philipp Loske

Institute of Theoretical Physics, Technische Universität Berlin, Hardenbergstraße 36, 10623 Berlin, Germany

Leon Merfort

Institute of Theoretical Physics, Technische Universität Berlin, Hardenbergstraße 36, 10623 Berlin, Germany

Vesna Vuksanović

Aberdeen Biomedical Imaging Centre, University of Aberdeen, Lilan Sutton Building, Foresterhill, Aberdeen AB25 2ZD, UK

E-mail: vesna.vuksanovic@abdn.ac.uk

*These authors contributed equally. 
12 data with numerical simulations of brain interactions. In this paper, we present a

13 summary of a data-driven computational model of synchronization between distant

14 cortical areas that share a large number of overlapping neighboring (anatomical)

15 connections. Such connections are derived from in-vivo measures of brain connec-

16 tivity using diffusion-weighted magnetic resonance imaging and are additionally

17 informed by the presence of significant resting-state functionally correlated links

18 between the areas involved. The dynamical processes of brain regions are simu-

19 lated by a combination of coupled oscillator systems and a hemodynamic response

20 model. The coupled oscillatory systems are represented by the Kuramoto phase os-

${ }_{21}$ cillators, thus modeling phase synchrony between regional activities. The focus of

22 this modeling approach is to characterize topological properties of functional brain

23 correlation related to synchronization of the regional neural activity. The proposed

24 model is able to reproduce remote synchronization between brain regions reaching

25 reasonable agreement with the experimental functional connectivities. We show

26 that the best agreement between model and experimental data is reached for dy-

27 namical states that exhibit a balance of synchrony and variations in synchrony

28 providing the integration of activity between distant brain regions.

\section{$29 \quad 1$ Introduction}

30 Decoding the fundamental mechanisms underlying large-scale brain integration is

31 one of the major challenges of neuroscience. A dominant hypothesis states that

32 phase synchronization plays an important role for the integration of the neural

33 activities between distant sites of the brain. The interaction among distributed

34 brain regions through phase synchronization may form the basis for cognitive 
35 processing [1-3]. An increasing number of literature aims to establish a framework

36 of models designed to deal with this issue by means of shaping patterns of the

37 large-scale functional connectivity map [4-8].

$38 \quad$ In this paper, we discuss neural synchronization using simple concepts of oscil-

39 lators' dynamics [9]. To this purpose, we review a data-driven approach that uses

40 a network of Kuramoto models to simulate phase synchrony in the brain at rest

$41 \quad[10-12]$. This is one of the models that aim to recover the interplay between brain

42 structural and functional connectivity from the perspective of coupled oscillatory

43 processes [13-16]. This model shows that remote synchronization observed in the

44 brain at rest may be sustained by the shape of structural connectivity and simple

45 dynamical rules.

46 There is evidence that brain integrative functions cannot be fully predicted

47 from the anatomical structure $[4,7]$. Subsequently, one can argue that the dynam-

48 ics of information on top of structural connections enables the communication

49 between segregated brain areas. Kuramoto phase oscillator models have been used to explore fundamental mechanisms underlying the nature of this communication.

51 The basic idea is to incorporate topological properties of the large-scale brain 52 connectivity in the coupling structure of the model. These properties are usu-

53 ally derived from white-matter tractography. The model that we here present also

54 takes into account the functional connectivity map and transmission delays based

55 on realistic distances to help to focus on connections relevant for the brain state

56 under consideration.

57 Within this framework, dynamical models of the resting brain based on the

58 Kuramoto phase oscillators have been able to shed light on how (i) the resting-

59 state brain activity emerges from a sufficient degree of noise and time delays [13, 
81 The brain is a complex dynamical system characterized by nonlinear interactions

14], (ii) relay-like interactions between distant brain areas emerge from modular network structures [11], and (iii) the anatomical hubs in the brain synchronize their activity [17]. A similar approach can be utilized to study pathological states due to the epilepsy [7], stroke [18] or schizophrenia [19]. An additional common feature of these models is the presence of variations in network synchrony, which is indicative of network metastability. This dynamical property allows for flexible changes of the network synchrony, i.e., partial and time-varying synchronization of neural activity across regions. These partial synchronization patterns in neural networks induce fluctuations at the level of synchrony of sub-networks leading to correlated fluctuations in low-frequency activity present in functional magnetic resonance imaging (fMRI) time series $[13,17,20]$.

This paper is organized as follows: In section 2, we first introduce the concept of brain networks, which can be studied using methods from graph theory. We then continue by describing nonlinear dynamics principles behind synchronization models and their application on neural dynamics (section 3). In section 4, we investigate the role that synchrony and its variations play in brain activity based on simulated neural/blood oxygenation level-dependent time series. We also provide new findings that combine different approaches used in previous studies. We conclude in section 5 with a brief summary, consider model limitations, and suggest further studies.

\section{Brain networks and neuroimaging data}

82 and emergent behaviors. This description - today nearly a consensus among neu- 
roscientists - contrasts the approach of brain functional specialization, a concept widespread until the early 20th century [21]. A common basis of both viewpoints

85 is the hypothesis that every mental state is connected to a physical brain state.

86 This hypothesis is known as a neural correlate [22]. The functional specialization

87 approach has triggered considerable contributions to neuroscience. Nevertheless,

88 it faces serious limitations, mainly when employed to investigate high-level cog-

89 nitive functions. On the other hand, the complex system approach has been very

90 promising for such investigations. In short, the focus from the first to the latter

91 approach has been shifted from where the function takes place to how the function

92 takes place in the brain [23].

The popularization of the idea of the brain as a complex dynamical system was especially promoted by the recent development of noninvasive imaging technologies that were able to record the time-dependent activity in the human brain as a whole [24]. Among those technologies, functional magnetic resonance imaging (fMRI) played a particularly important role. Roughly speaking, the data recorded via those functional neuroimage techniques consist of temporal series associated with linear and nonlinear functional relationships between brain regions and are understood as a proxy for neural activity. These series are recorded from collective signals of neural populations that form synchronized local circuits. The current challenge is to unveil the rules behind global brain activity and how they are connected to the range of cognitive states. 
(a)

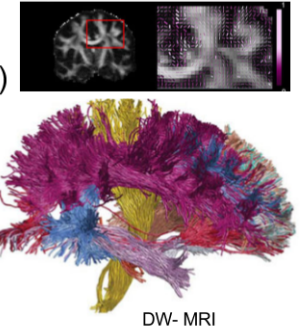

(d)

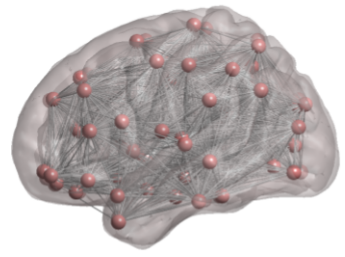

Graphical representation

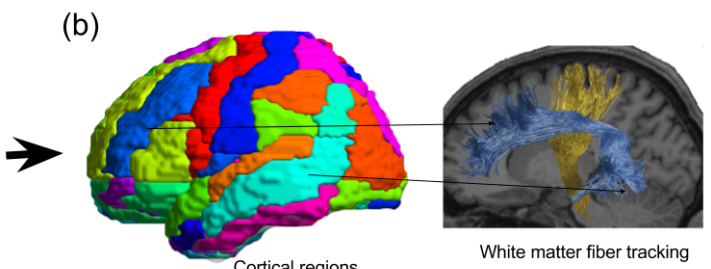

(c)

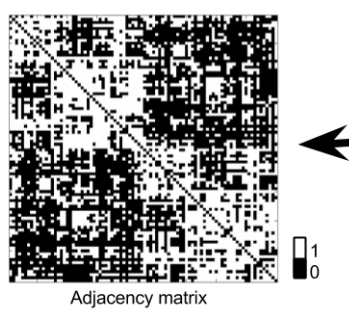

Adjacency matrix

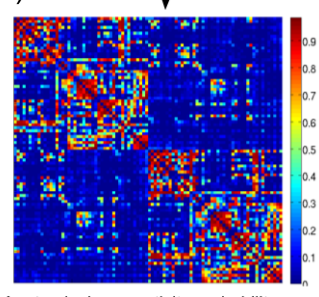

Anatomical connectivity probability map

Fig. 1 Anatomical network. (a) Diffusion-weighted magnetic resonance imaging (DW-MRI) and artistic reconstruction showing the fiber tracts. (b) Parcellation according to a cortical anatomical atlas and density of tracts between two pairs of areas. (c) Matrix of the anatomical connectivity probability of structural connections between pairs of regions. (d) Network construction: the adjacency matrix obtained by thresholding and the corresponding structural brain network. Sources: The DW-MRI figure and its artistic reconstruction is a reproduction of reference [25]. The brain images and network were created with the help of BrainNet Viewer [26]. The data for the anatomical connectivity probability from reference [27].

\subsection{Graph theory and brain connectivity maps}

Graph theory or network science is a novel way to study topology of the structural and functional organization of the brain which consists of describing it in terms of nodes (brain regions) and edges (the structural connections or functional relationships). Before we discuss how to define brain connectivity using graph-theoretical concepts, it is important to clarify the distinction between two different types of large-scale brain connectivity frequently mentioned in the literature.

The anatomical connectivity map is the map of structural connections between brain regions [28]. This network is stable on shorter timescales, but it may change over larger times due to neuronal plasticity [23]. The classical way to map structural connectivity is tracing neuronal paths by means of invasive and postmortem 
methods [29]. Due to this fact, it cannot be used to create a large dataset of the human brain. Alternatives come with the advance of neuroimage techniques, such as diffusion-weighted magnetic resonance imaging (DW-MRI), where anatomical fibers may be inferred by means of statistical models. Such methods allow in-vivo tractography of white-matter fibers. See references [30-32] for details about structural connectivity and how to acquire it from the human brain. Figure 1 depicts a schematic illustration of the workflow to extract a brain graph from imaging data. In short, the adjacency matrix is obtained from the anatomical connectivity 123 probability map by thresholding, that is only probabilities above a threshold result in a link in the brain graph. 


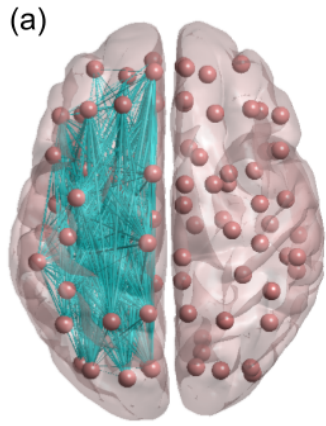

(b)
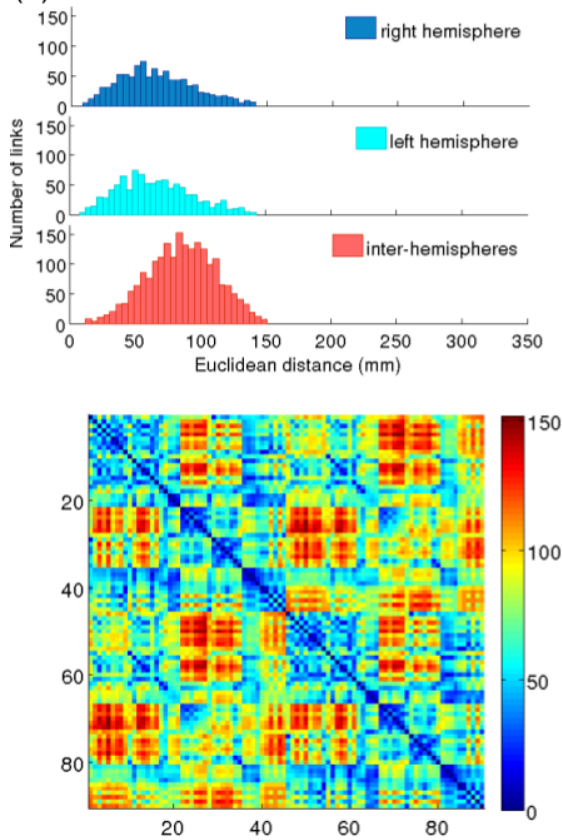
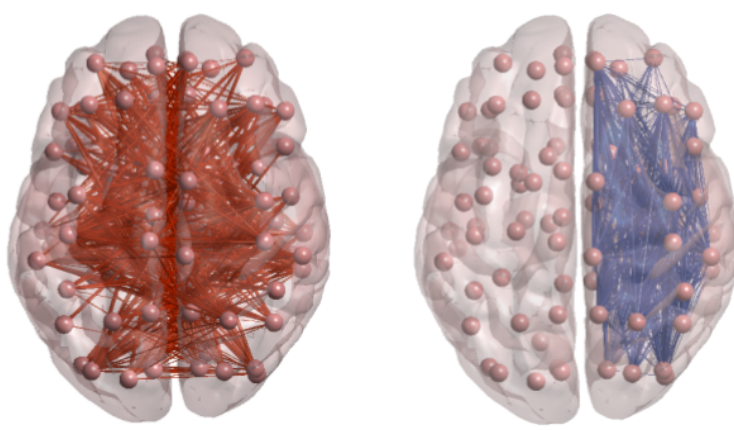

(c)
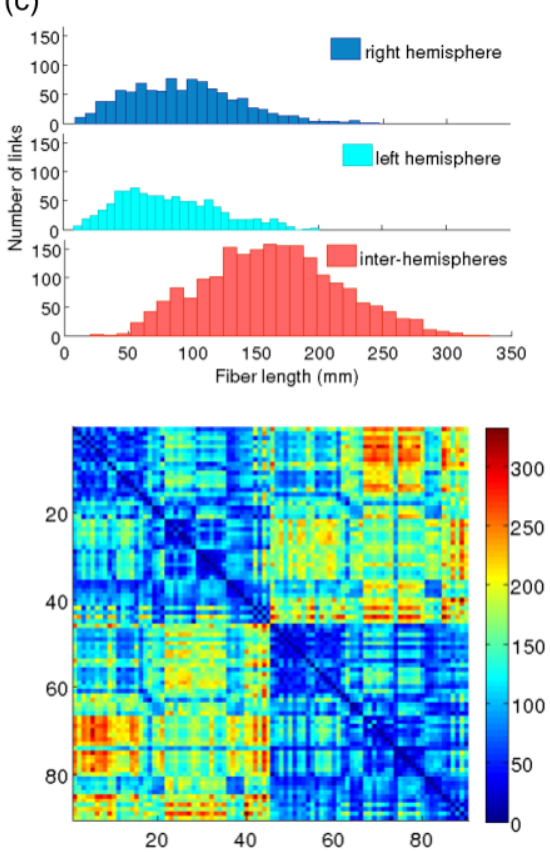

Fig. 2 Euclidean distances and fiber lengths. (a) Representation of networks, that is 90 brain regions according to the Automated Anatomical Labeling (AAL) parcellation [33] as nodes connected by links in the left hemisphere, between hemispheres, and in the right hemisphere respectively. (b) Top: Histograms of Euclidean distances in the right (blue), left (cyan), and between (red) hemispheres. Bottom: Matrix of the Euclidean distances between pairs of cortical regions. (c) Top: Histograms of the fiber lengths in the right, left, and between hemispheres. Bottom: Matrix of the fiber lengths between pairs of cortical regions. The data of the fiber lengths were taken from reference [27]. The brain networks were created with help of BrainNet Viewer [26]. 


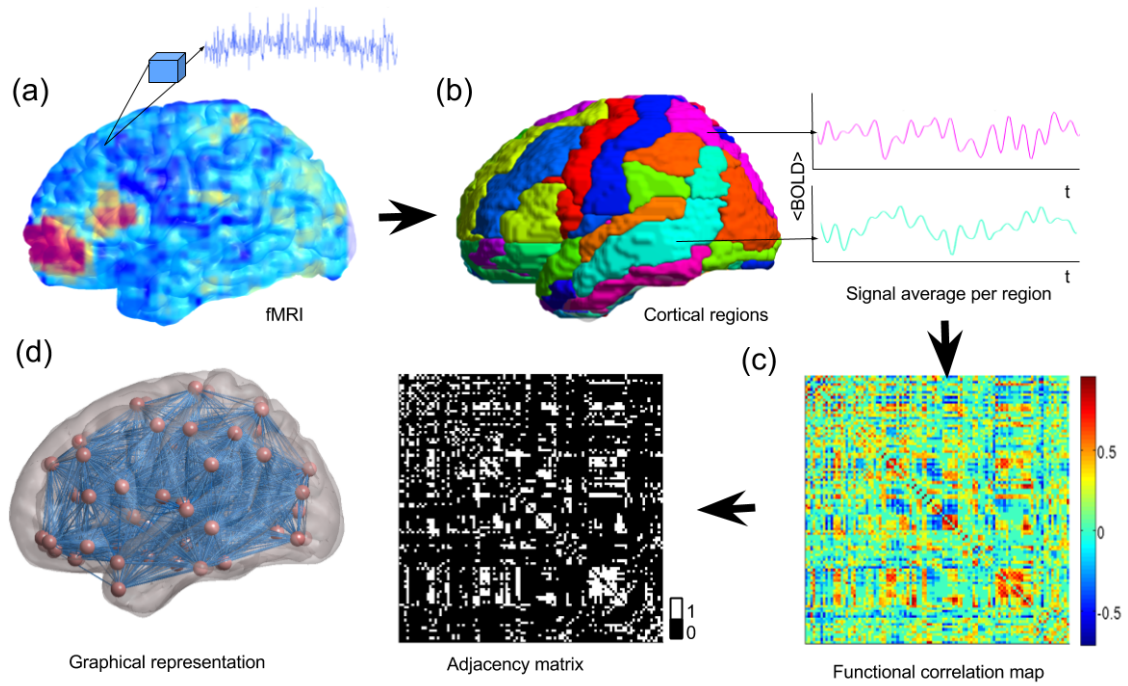

Fig. 3 Functional network. (a) Functional magnetic resonance imaging (fMRI) and bloodoxygen-level-dependent (BOLD) signals recorded for each voxel. (b) Parcellation according to cortical anatomical atlas and the averages of the signals from two regions. (c) Functional correlation between BOLD time series for every pair of regions. (d) Network construction: the adjacency matrix obtained by thresholding and the corresponding functional brain network. The brain images and network were created with the help of BrainNet Viewer [26].

The procedure of DW-MRI leads to an unexpected result. In order to quantify the probability, with which two brain regions of interest are structurally connected, one constructs a three-dimensional trajectory of the fiber tract between the centers of those regions. This provides a gateway to measure the length of the connection. Figure 2 depicts the distribution and distance matrices of these fiber lengths in panel (c). Compared to a naive estimate based on the Euclidean distance between regions considered in the Automated Anatomical Labeling (AAL, see reference [33]) shown in panel (b), one can see that the distributions of intraand inter-hemispheric connections exhibit qualitatively the same shape and that the fiber lengths stretch to larger values. As it will be explained in detail in section 3.2, this distance can be used to approach transmission delays between the brain regions. 
Functional relationships in the brain are usually described in the form of so called functional connectivity maps. They map the temporal correlations between regional activities [35], whose modular-like organization supports resting state networks as well as cognitive and behavioral functions. Therefore, they refer to a functional relationship irrespective of whether or not there exist anatomical connections. Functional connectivities are derived from time traces obtained by recordings of variations in the blood-oxygen-level-dependent signal (BOLD signal) due to brain activity. For a schematic depiction of the generation of functional connectivity maps, see figure 3 . In this work, we are interested in simulating the functional connectivity based on networks obtained from neuroimaging data. In the following, we briefly describe how a functional connectivity map, or functional network, can be obtained from fMRI data using graph theory.

The fMRI data is a 3-dimensional image of the brain acquired over time. At the finest spatial resolution of such an image, each voxel (typically of size 1-2 $\mathrm{mm}^{3}$ ) gives rise to one time series. For a large-scale analysis of the whole brain, the functional network may be defined as follows: The graph nodes represent regions of interest, usually defined by cortical regions obtained by parcellating the voxels in the fMRI measurement according to a cortical brain atlas [33,34]. Each of the resulting regions of interest, that is nodes in the brain network, gives rise to one time series that represents the BOLD signal in this region. Usually, this series is obtained by averaging over the respective set of voxels. Subsequently, network links are defined on the basis of a correlation between time series from each pair of regions of interest, which yields a weighted coupled network, indicating the similarity in the activities of the respective nodes. These maps connect brain regions irrespective of the presence of actual anatomical links. It is worth mentioning that 
fMRI captures the variation in the BOLD signal, that is, it is an indirect measurement of neural activity and includes several confounders [36]. Before constructing functional networks, the data undergoes a number of pre-processing steps, e.g., for motion correction, to remove spurious information, and band-pass filtering to improve the signal-to-noise ratio. For further details about data pre-processing, see references [11,37-39]. For more details about networks from fMRI data, see references $[40-43]$.

One can describe functional networks by an adjacency matrix $\left\{A_{i j}\right\}_{i, j=1, \ldots, N}$, in which each matrix element takes the value of unity if a pair of nodes is connected and zero otherwise. The pair of nodes is considered to be connected when the respective entry in the correlation matrix exceeds a predefined threshold value. There are different methods used to threshold the matrix and to retain only those values which are statistically significant. The value of the threshold has a direct influence on the network density [41]: the higher the threshold, the lower the network density. By defining its adjacency matrix and thus selecting the network topology, it is possible to detect universal behaviors of coupled dynamical systems such as synchronization or metastability. One can also consider weighted instead of binarized matrices. The weight can be add to the model by consider some information from experimental data. For example, it can be proportional to the density of fiber tracts between the two cortical regions [44]. In the current approach, however, we aim for simplicity of the model by considering only anatomically relevant connections of higher probability. For a detailed overview of complex brain networks, see reference [45]. 
2.2 Spontaneous synchronicity and resting state brain networks

Most of the early neuroimage analyses were designed to test the hypothesis of localized functional brain specificity. The goal was to investigate, which region in the brain is activated during a specific task. This design is rooted in neuroanatomists' concepts of the 18th century and was largely discussed at the end of the 20th century [21]. In fact, several experiments had supported the paradigm that specific brain regions are correlated with specific functions, especially basic sensory and motor tasks [21]. However, the functional specificity started to receive relevant critical remarks. This reductionist approach could not explain high-level cognitive processes such as emotions, creativity, and consciousness.

In the middle of the 1990's, a new insight changed the focus of research and transformed prior knowledge. It was recognized that there are large-scale synchronization patterns in the spontaneous fluctuation of brain activities in the absence of external input [46]. Non-random patterns were observed in the data scanned from subjects in the resting state, that is lying down in the absence of tasks or attention demands. These findings were corroborated and complemented by several studies using different neuroimaging techniques [47]. Further descriptions of these patterns, termed as resting state networks (RSN), can be found in references [48, 49]. The discovery of the RSN is considered a milestone in contemporary neuroscience for different reasons. It supports the regard of the brain as a dynamical complex system. The detection of large-scale patterns for resting state conditions reflects the existence of coordinated intrinsic dynamics. This spontaneous interregional synchronization indicates self-organized capability. On one hand, it has been suggested that RSN are related to high-level brain functions such as inter- 
nal mental processes and consciousness. This hypothesis is supported by studies that show variations in statistical features of RSN in altered states of consciousness [50-52] and mental disorders such as autism [53] or schizophrenia [54]. On the other hand, RSN have also been detected in people subjected to deep sedation [55], sleep [56], coma [57], or even vegetative states [58]. This fact could, in principle, challenge the hypothesis of RSN as a signature of consciousness. However, Barttfeld et al. show that RSN in monkey brains under deep anesthesia are more strongly correlated to the anatomical connectivity map in comparison to regular RSN in a resting state of wakefulness [59]. They show that in the case of loss of consciousness, the functional activity is tied to anatomical connectivity.

Their study is in agreement with hypotheses made in previous theoretical works $[5,60]$. Functional networks in resting states where the subject is awake are characterized by long-range synchronicity and high variability of patterns. It had been observed that an anatomically connected pair of nodes has a high probability to be functionally connected. However, functional connectivity is frequently observed between brain regions without direct structural links [5,61]. The understanding of the rules that allow both long-range synchronization and flexibility of patterns on functional networks may be the key to decrypt the mechanisms behind high-level brain functions. Models using dynamical systems, e.g., oscillator models, are the most promising tools to tackle this challenge.

\section{Brain activity and synchronization models}

In this section, we build a bridge between nonlinear dynamics and computational neuroscience. At first, we summarize the concept of synchronization and then 
develop a simple mathematical model that will be used in section 4 . We also briefly elaborate, how a BOLD signal can be inferred from a neural time series by means of the Balloon-Windkessel model.

3.1 Nonlinear dynamics and synchronization in the brain

Synchronization plays an important role in various contexts including physics, biology, and beyond [9,62-65]. In neuroscience, some forms of cooperative dynamics have been associated with pathological states like migraine, Parkinson's disease, or epilepsy [66-76]. Besides these detrimental forms of synchrony, it is also considered a crucial mechanism for recognition, learning, and processing of neural information.

In general, neuronal systems can be described by physiological models such as the Hodgkin-Huxley equations [77]. These type of models account for many physiological details and processes. Accordingly, they offer a detailed description of a single cell. On the downside, they often consist of many equations and many parameters and their applicability on large ensembles of elements is highly questionable, which also holds for a bifurcation analysis.

On the other side of the spectrum of complexity, there are normal-form equations. These phenomenological models capture the main dynamical behavior of neurons such as the type of excitability and can be coupled together in large networks with reasonable numerical effort. In some cases like the FitzHugh-Nagumo model $[78,79]$, they can be derived as low-dimensional approximations, which are better suited for a bifurcation analysis, because they contain only a few parameters and nonlinearities. The price that one has to pay is a vague - at best qualitative - 
correspondence to physiological quantities like membrane potential, ionic currents, etc.

Self-organized dynamics of brain regions into functional networks often follow the underlying structural connections. There are, however, functional correlations between cortical regions that are not directly connected. Thus, the mechanisms for functional connectivity between distant cortical regions are still subject to intense research efforts. For example, indirect connections can support collective dynamical behavior on the brain network and pronounced pair-wise correlation of brain regions. If such indirect connections are involved, that is, there is no direct anatomical link between highly-correlated regions, the dynamical pattern can be called remote synchronization $[80,82]$. The amount of synchrony depends on properties of the coupling topology such as the symmetry of interactions $[82$, $83]$.

3.2 The Kuramoto model of phase oscillators

Neural activity evolves through brain networks as a dynamical process, which can be approximated by either neural fields [84] or neural models [85]. To simulate the dynamical behavior of such processes, one can also choose the even simpler, that is less complex, model of Kuramoto-like phase oscillators [11-13,16], which has been established as a general model for oscillatory dynamics.

The classic Kuramoto model consists of dynamical equations with one phase variable for each network node [86]. The nodes are connected in an all-to-all topology and the interactions are mediated by sinusoidal functions of the phase differ- 
ences of all pairs of oscillators:

$$
\dot{\phi}_{i}=\omega_{i}+\frac{K}{N} \sum_{j=1}^{N} \sin \left[\phi_{j}(t)-\phi_{i}(t)\right], \quad i=1, \ldots, N,
$$

where $K$ is a global coupling strength. The parameter $\omega_{i}$ denotes the natural frequency of the i-th oscillator drawn from a given distribution. For reviews on the relevance and universal applicability of the Kuramoto model see references [87, $88]$.

In order to analyze the amount of synchrony in the network, the global order parameter, which is given by the center of mass of phase variables of each node distributed on the unit circle, has proven to be very insightful:

$$
R(t)=\left|\left\langle e^{i \phi_{k}(t)}\right\rangle_{N}\right|, \quad k=1, \cdots, N,
$$

where $\langle\cdot\rangle_{N}$ denotes the average over all nodes in the network. The order parameter can easily be applied to the simulated time series of neural activity $[13,89$, 91]. Then, its temporal mean value $\langle R(t)\rangle$ and standard deviation provide information about the level and temporal fluctuations of synchrony. The latter can be interpreted as metastability as discussed below. It is easy to see that in equation $(2), R(t)$ tends to zero, if the phase variables are dispersed across phase space, that is, when they are highly desynchronized. In the opposite case, when most of oscillators have close phase variables, one obtains the limit $R(t) \longrightarrow 1$.

In general, the number of phase variables that become locked and synchronized, depends on the coupling strength $K$. This quantity can be used as a control parameter to study emerging patterns of synchrony. For a given natural frequency distribution, there is a threshold or critical coupling strength $K_{c}$ above which the 
coupled system starts to synchronize. This observation can be described as a phase transition. Results based on the global order parameter defined in equation (2) can be seen as a mean-field approach, that is, the simplest case of isotropic interaction.

To study neuro-biological systems, it is necessary to consider inhomogeneities of the coupling topology connected to a variety of different complex networks. In addition, one can investigate the influence of time delay in the coupling term. Then, equation (1) can be extended as follows

$$
\dot{\phi}_{i}=\omega_{i}+C \sum_{j=1}^{N} A_{i j} \sin \left[\phi_{j}\left(t-\tau_{i j}\right)-\phi_{i}(t)\right], \quad i=1, \ldots, N,
$$

where the coupling strength is denoted by $C$. Now, structural inhomogeneities can be accounted for by pair-wise transmission delays $\tau_{i j}$ in the coupling term. This makes network interactions biologically more plausible $[92,81]$ and prevents full synchronization of the network $[82,93]$. The delays are inferred from the distance $\Delta_{i j}$ between nodes $i$ and $j: \tau_{i j}=\Delta_{i j} / v$ with a signal propagation velocity $v$ in the range of $1 \mathrm{~m} / \mathrm{s}$ to $20 \mathrm{~m} / \mathrm{s}$. Alternatively, one can introduce link-dependent phase offsets in the coupling term [94]. Less pronounced synchronization can be interpreted as a preferred dynamical state and an important property of the neural networks, as fully synchronized brain dynamics are never observed experimentally. From the results of models of the resting-state dynamics, for instance, it has been argued that the brain operates in so-called metastable states and never reaches full synchronization $[14,95]$.

The network matrix $\left\{A_{i j}\right\}$ defines the interactions between the neural processes. As elaborated in section 2, one can construct this matrix using empirically derived structural connectivity: the non-zeros entries of the matrix correspond to 
existing connections between respective brain regions. Alternatively, one could also generate an adjacency matrix based on the functional connectivity. Further details on the applied procedure, which uses a combination of anatomical and functional connectivity maps, will be discussed in section 4 below. See also figure 4 .

3.3 Inferring BOLD signals: the Balloon-Windkessel model

As mentioned in section 2.1, functional connectivity maps are networks of brain regions that are based on a statistical dependence between fMRI time series $[15$, $46,96]$. The underlying time series of BOLD activity are a function of changes in cerebral blood flow, cerebral blood volume, and cerebral metabolic rate of oxygen consumption and typically exhibit significant correlations for frequencies below $0.1 \mathrm{~Hz}$ in the resting state [46]. In order to compare the numerically obtained neuronal activity with the empirical BOLD signal, we make use of the BalloonWindkessel model [97], which has been established in many computational studies of the resting-state brain activity. Briefly summarized, this model considers the neuronal time series as an input signal [98] and computes the hemodynamic response, which can then be related to the BOLD signal. Since the neuronal activity and the blood response operate on different time scales of milliseconds and seconds, respectively, the Balloon-Windkessel model acts as a low-pass filter on the high-frequency neuronal signal. To allow for comparison with the experimentally measured BOLD signal, we match a simulation's duration to the lengths of the experimental recording. 
4 Data-inspired models: from neuroimage information to brain activity models

From a modeling perspective, the observed spatio-temporal patterns in brain activity are shaped by the complex relationship between the dynamics of individual oscillators and global synchronization [99]. As described in section 3.2, these competing dynamics can be characterized by the amount of synchrony in the network and its variations over time. The latter indicates dynamical metastability. It has been suggested that these variations of the network synchrony shape the patterns of coordinated activity between brain regions and thus, enabling dynamical exploration of different network configurations $[44,89,100]$. Such functional network configurations are constrained by the underlying anatomical structure [101] - another key ingredient of the model.

Anatomical brain connections enter models of the brain dynamics in the form of the coupling matrix, whose elements represent actual neural paths between brain regions - network nodes - as described in section 2.1. The topology of this matrix is usually static, i.e., the number of links between the nodes is preserved. Figure 4 provides a schematic diagram of the model workflow. A combination of experimental anatomical and functional connectivity maps leads to an adjacency matrix that defines the interaction of the oscillators in the simulations. A link is present if it is anatomically justified and has a high probability to have functional connectivity, which is implemented as an element-wise multiplication of binarized anatomical and functional connectivity matrices. By averaging and binarizing the connectivity matrices one can select the connections between pairs of regions with higher statistical probability, considering all subjects. Since the functional connectivity 


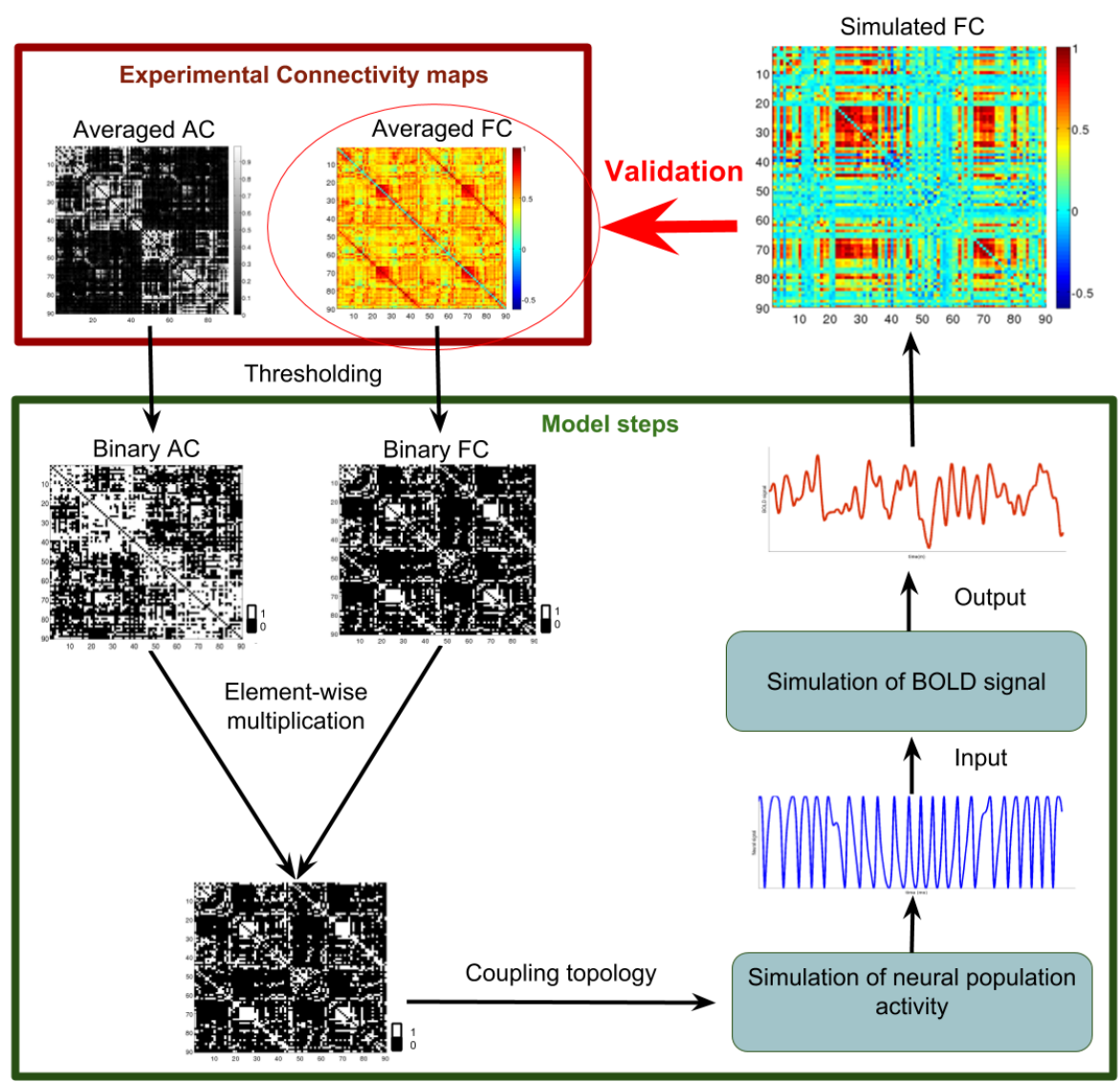

Fig. 4 Schematic diagram for the modeling framework. Anatomical connectivity (AC) and functional connectivity (FC) maps extracted from DW-MRI and fMRI as group averages over 26 subjects, respectively, are binarized and combined to compute the adjacency matrix that provides the coupling topology in the simulations. Neural population activity is simulated and used as input to infer the simulated BOLD signal. The resulting time series of each node are correlated pair-wise leading to a simulated functional connectivity matrix, which is compared with the experimental functional connectivity map.

map has been derived from resting-state data, the element-wise multiplication se-

lects those anatomical connections that directly connect brain regions that tend to be highly correlated in this condition. This step is important to evaluate the first level influence of anatomical connections in the remote synchronization of brain regions activities. 

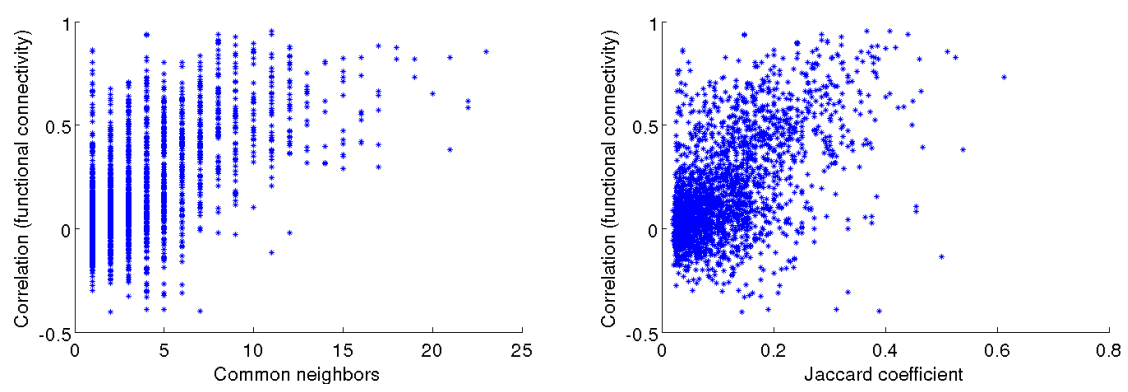

Fig. 5 Functional connectivity between pairs of network nodes, i.e., regions of interest, which are not directly connected in the considered brain graph, as a function of the number of common neighbors (left) and Jaccard coefficient (right). Parameters in the simulation of equation (3) with delays calculated from the fiber lengths: threshold for functional connectivity in the network generation $r=0.56$, coupling strength $C=54$, and signal transmission velocity $v=5 \mathrm{~m} / \mathrm{s}$.

We use this approach to derive the coupling topology for our simulations as our primary aim is to reconstruct long-distance functional correlations that emerge from the underlying anatomical paths. Previous works have used this model to explore the contribution of the long-distance functional interactions - those that are not supported by direct neural paths - to the brain functional correlations in the resting-state activity $[11,12]$. These works have shown that the integration of the brain functions may arise from relay-like phase interactions between neural oscillators that share large parts of their individual network's neighborhood. In this review, we present additional analyses based on brain dynamics that include time delays in the phase interactions between the neural oscillators, as given in equation (3). The time-delayed interactions are determined by the empirical length of the connections between the regions. See figure 2. It is worth mentioning that the time delays on the real brain may be affected by heterogeneities related to local physiology. For example, the velocity of signal transmission depends on other biological aspects such as myelination and axon thickness. The model in this paper 
A

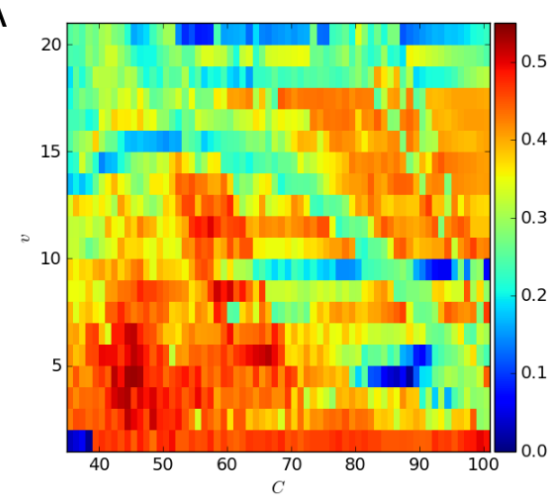

B

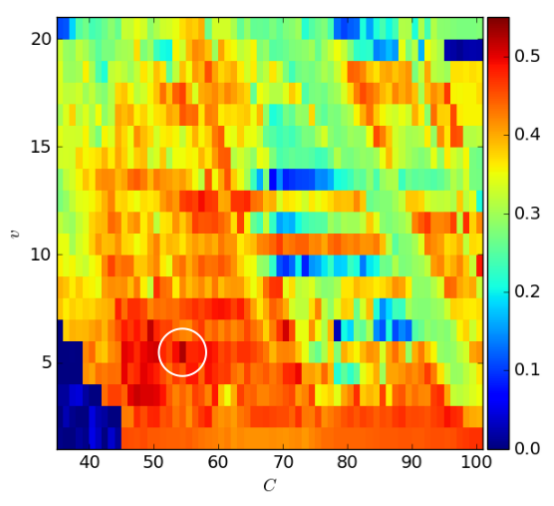

Fig. 6 Pearson correlation coefficient between experimentally derived and simulated functional connectivity in the parameter space spanned by coupling strength $C$ and signal transmission velocity $v$. The simulations are based on equation (3) with time delays calculated from the Euclidean distances and lengths of fiber tracks between regions of interest in panels $\mathrm{A}$ and $\mathrm{B}$, respectively. See figure 2 for further information on the distances. The white circle in panel $\mathrm{B}$ marks the $(C, v)$-values used in figures 5 and 7 with a maximum Pearson correlation of 0.53.

accounts for the influence of time delay by (i) considering the heterogeneity of distances and (ii) assuming a fixed velocity.

Figure 5 shows the effect of remote synchronization. It depicts the functional connectivity for any pair of nodes $i$ and $j$ that do not share a direct connection according to the coupling matrix in dependence on the number of common neighbors and the relative overlap of the neighborhoods $N_{i}$ and $N_{j}$. The latter is quantified by the Jaccard coefficient

$$
J_{i j}=\frac{\left|N_{i} \cap N_{j}\right|}{\left|N_{i} \cup N_{j}\right|}
$$

where $\left|N_{i}\right|$ denotes the number of neighbors of node $i$, that is, its degree. In words, $J_{i j}$ is the relative size of the intersection between the two node sets with respect to their union and takes values in the interval $[0,1]$ with the limit cases of zero and unity referring to no and perfect overlap, respectively. We observe an increase of functional connectivity as the overlap of neighborhoods becomes larger. This is in agreement with previous findings $[11,12]$. 


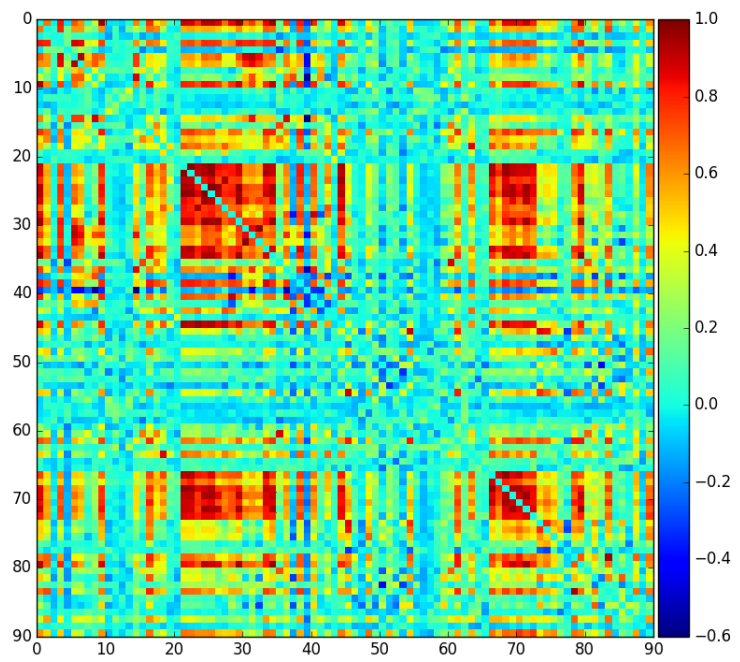

Fig. 7 Exemplary, simulated functional connectivity based on equation (3) with time delays calculated from the fiber lengths between regions of interest (cf. figure 2). Parameters: $C=54$ and $v=5 \mathrm{~m} / \mathrm{s}$.

A systematic exploration of the parameter space spanned by coupling strength $C$ and signal transmission velocity $v$ is depicted in figure 6 , where the left and right panels refer to time delays in equation (3) according to the Euclidean distances and lengths of fiber tracks between brain network nodes, respectively. Recall that the finite velocity is the cause of delayed interactions. The color code indicates the agreement with the experimentally derived and simulated functional connectivity quantified by the Pearson correlation coefficient. Overall, the results of the two panels in figure 6 are qualitatively very similar. Note that a rescaling in the $v$-direction would lead to a quantitative agreement that could be explained by the shape of the distance distributions shown in figure 2. Larger velocities could compensate for the shorter distances. According to our analysis, the Euclidean distance between different brain regions - with a proper scaling factor - can be used 


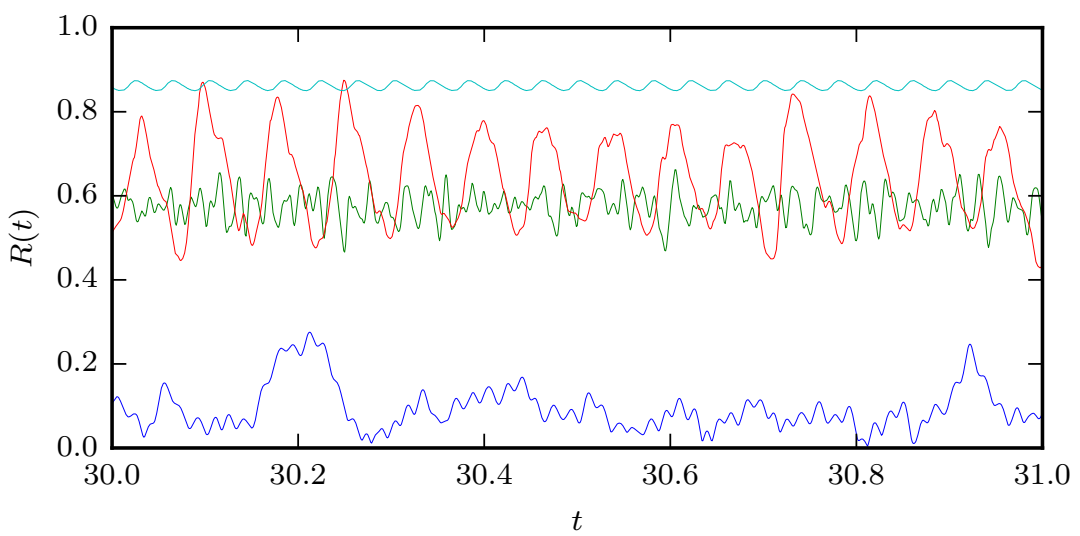

Fig. 8 Global order parameter defined in equation (2) for different signal transmission velocities $v=0.1 \mathrm{~m} / \mathrm{s}$ (blue), $5 \mathrm{~m} / \mathrm{s}$ (green), $20 \mathrm{~m} / \mathrm{s}$ (red), and $100 \mathrm{~m} / \mathrm{s}$ (cyan). The coupling strength is fixed at $C=54$.

to account for finite signal transmission velocities along the neural connections.

The highest Pearson correlation is found in the range of plausible transmission velocities. For weak coupling, that is, low values of $C$, the interaction via the network is not strong enough to trigger significant self-organized synchrony in neural activity or BOLD signals.

The best agreement of the simulated functional connectivity with the experimental functional connectivity is observed for $C=54$ and $v=5 \mathrm{~m} / \mathrm{s}$. Figure 7 shows the corresponding functional connectivity matrix obtained from the simulations. One can see clusters of well-correlated nodes in the brain network.

Considering the form of the global order parameter $R$ given by equation (2) the particular parameter combination choice, $C=54$ and $v=5 \mathrm{~m} / \mathrm{s}$, is justified. The temporal average $\langle R(t)\rangle$ of the order parameter quantifies the average amount of synchrony in the brain network and its standard deviation can be used to inspect metastability. Figure 8 depicts the time series of $R$ for a fixed coupling strength $C=54$ and different velocities $v$. Large values of $v$ result in an almost 


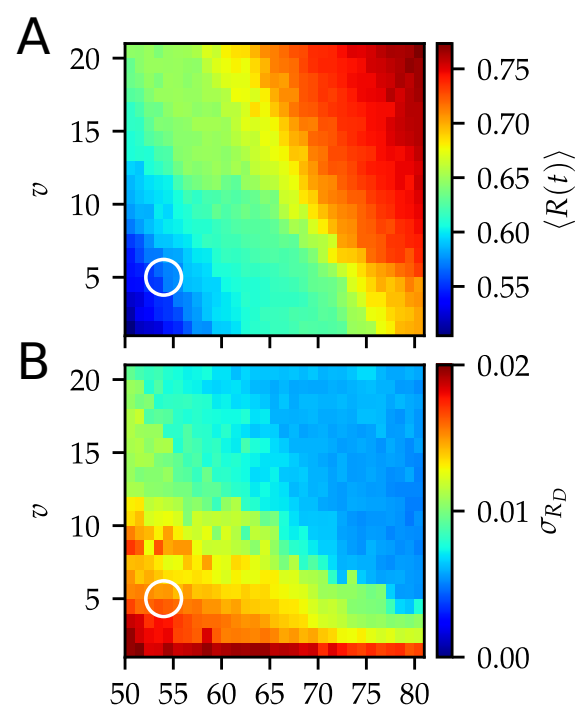

C

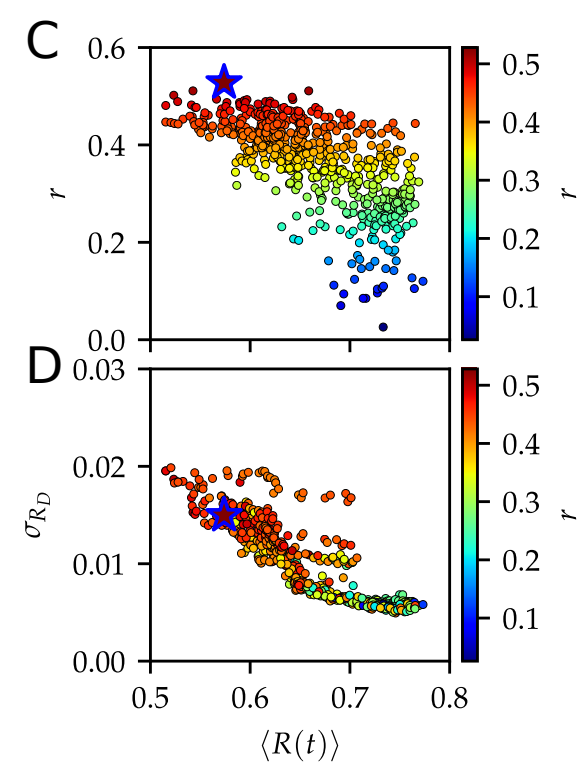

Fig. 9 Panels A and B: parameter scan of the average order parameter $\langle R\rangle$ and detrended fluctuations $\sigma_{R_{D}}$ as color code in the $(C, v)$-plane, respectively (cf. figure 6). Panels $\mathrm{C}$ and D: average order parameter $\langle R\rangle$ vs. Pearson correlation and detrended fluctuations $\sigma_{R_{D}}$, respectively. The color code refers to the Pearson correlation coefficient $r$ between experimental and simulated functional connectivity (cf. figure. 6). The white circles and blue star marks the values $C=54$ and $v=5 \mathrm{~m} / \mathrm{s}$ used in figures 5 and 7 with a maximum Pearson correlation of 0.53. The fit of the modeled functional correlations with the experimental data is best for a dynamical state that simultaneously balances synchrony and metastability.

instantaneous coupling, for which the coupling function in equation (3) supports

the emergence of robust synchronization. This is indicated by a high value of $R$ that

does not exhibit strong fluctuations around its mean (cyan curve, $v=100 \mathrm{~m} / \mathrm{s}$ ).

As velocities decrease, the order parameter becomes smaller, but still remains its periodicity (red curve, $v=20 \mathrm{~m} / \mathrm{s}$ ). In the range of plausible velocities (cf. green curve, $v=5 \mathrm{~m} / \mathrm{s}$ ), we find a balance between synchrony and metastability, that is, a reasonable value of $\langle R(t)\rangle$ together with seemingly random fluctuations. These observations are in agreement with our previous studies [11,12].

Figure 9 shows how functional interactions - high values of the correlation coefficient $r$ between the modeled and experimental dynamics - can be connected 
458 scribed in reference [27]. cha scribed in reference [27].

to a dynamical behavior that balances the synchrony $\langle R(t)\rangle$ and the variations in synchrony $\sigma_{R_{D}}$. Figures $9 \mathrm{~A}$ and $\mathrm{B}$ depict the dependence of the average order parameter $\langle R\rangle$ and its fluctuations $\sigma_{R_{D}}$ on the coupling strength $C$ and the transmission velocity $v$, respectively. For the fluctuations $\sigma_{R_{D}}$, we detrended the periodic behavior of $R(t)$ (cf. figure 8). This detrending removes the contributions to the standard deviation that do not reflect fluctuations in the dynamics. One can see that the good agreement with the experimental matrix is found in a region of the parameter space that presents some level of synchronization (panel A) and fluctuations (panel B). These dynamical conditions allow for the emergence of synchronization on the functional networks and also keep some level of flexibility for the emergence of different synchronized patterns over time. Figures 9C and D further corroborate this balance in the simulated, metastable dynamics. The values $C=54$ and $v=5 \mathrm{~m} / \mathrm{s}$, which lead the maximum Pearson correlation between simulated and experimental functional connectivities, are marked by white circles and a blue star. These findings are consistent with the previous simulations of task-free $[13,44]$ and task-dependent [89] brain activity, which are based on similar simplified models that take into account a few key parameters of structural and functional brain connectivity.

The experimental fMRI data sets used in this paper are available from the 1000 Functional Connectome Project website (http://fcon_1000.projects.nitrc.org/).

We consider functional scans from the Berlin Margulies data to calculate the group average. The data consist of open-eyes resting-state measurements of 26 subjects (ages: 23-44) [102]. For details on the pre-processing steps, see reference [11]. For the anatomical connectivity probability, we use DW-MRI data from a study de- 
Modern brain imaging methods allow for a quantitative study of both local activity dynamics and the interdependence between activities in anatomically distant cortical areas, which is known as functional connectivity. With this review, we have summarized one of many multidisciplinary approaches to model such functional interactions. Leveraging interdisciplinary theoretical techniques, inspired by complex system theory and applied mathematics, and existing experimental data from noninvasive brain imaging, the proposed modeling framework contributes to the development of viable analytical and modeling techniques leading to significant insight into dynamical mechanisms of the brain.

The particular model, which we consider in this review, combines experimental anatomical and functional connectivity between cortical regions to generate a network topology of the brain at rest. By varying the network interactions (using different coupling strengths and signal transmission velocities), it is possible to obtain correlation patterns in the simulated BOLD fMRI time series that are in agreement with experiments. We have shown that the model leads to the best agreement for a dynamical state that exhibits a balance between synchrony and temporal variations in synchrony. The proposed model allows to investigate the role of network structure and in particular indirect connections between distant cortical regions and to explore functional connectivity in the brain using numerical simulations of delay-coupled phase oscillators. For example, we have found higher functional connectivity, if the neighborhoods of respective nodes show a greater overlap. We have also compared the influence of time delay considering fiber track lengths and Euclidean distances between brain regions. We have observed no qual- 
itative difference in the simulations. This means that Euclidean distances - after rescaling - may be used to account for realistic coupling delays.

The procedure can easily be extended to a much larger field of brain states. For example, one can alter the adjacency matrix of the task-negative system by increasing the weights of connections between task-related nodes above unity, simulating a greater statistical relevance within the task-evoked state. Additionally, this procedure might give some insight into the brain shifting from the resting-state to task-evoked states and back.

The flexibility of the network topology generating process also gives an opportunity to manipulate node connections to adapt to neural activity observed in fMRI measurements of patients suffering from various brain disorders. Indeed, similar data-driven models had contributed to understanding some mechanisms of brain disorders $[103,7,90,91]$.

The limitation of this model is given by its purpose, which was to provide explanations for mechanisms generating coordinated activity between spatially distant brain regions. We focus our computations on how these long-distance correlations arise from realistic functional interactions, i.e. those that are also supported by direct structural connections. Thus, our model does not consider the role of coupling topologies that correspond directly to structural connectivity data. Models based on these structural connectivity topologies have been explored extensively in several studies (see references $[13,89,91]$ ), reaching - similarly to our model to an agreement with the experimental data only to a certain extent.

The model presented in this paper does not strive to give an accurate representation of the physiologically realistic brain activity. A much more physiologically based approach is needed to achieve a full understanding of the relation between 
experimental fMRI data and simulated neural activity. However, this goes beyond the scope of the main focus of the present work, that discusses a specific approach to find a simple way to simulate neural time series and to transform them into data, which can be compared to experimental fMRI measurements. This simplification is also adopted in similar studies found in references $[13,44,91,95]$. The model that we presented in this review can be extended in various way to incorporate more physiological details such as heterogeneities in the signal transmission velocities accounting for myelination or axon thickness. In addition, link weights can be introduced in the coupling matrix to include more information from experimental data.

The studies summarized in this article contribute to a better understanding of the relationship between complex brain networks and temporal dynamics of brain activity. They might also serve as a starting point to investigate brain network reconfigurations providing a modeling framework to explore transient, dynamical interactions, which enable diverse cognitive functions.

\footnotetext{
Acknowledgements AV and PH acknowledge support by Deutsche Forschungsgemeinschaft under grant no. HO4695/3-1 and within the framework of Collaborative Research Center 910. We thank Yasser Iturria-Medina for sharing the DW-MRI data including fiber lengths used in the study. We also thank Jason Bassett for helpful discussions.
}

\section{References}

1. T. Womelsdorf, J.M. Schoffelen, R. Oostenveld, W. Singer, R. Desimone, Science 316(5831), 1609 (2007).

2. P. Uhlhaas, G. Pipa, B. Lima, L. Melloni, S. Neuenschwander, D. Nikolic, W. Singer, Front. Integr. Neurosci. 3, 17 (2009). 
3. M. Bola, B.A. Sabel, NeuroImage 114, 398 (2015).

4. C.J. Honey, O. Sporns, L. Cammoun, X. Gigandet, J.P. Thiran, R. Meuli, P. Hagmann, Proc. Natl. Acad. Sci. U.S.A. 106(6), 2035 (2009).

5. G. Deco, V.K. Jirsa, A.R. McIntosh, Nat. Rev. Neurosci. 12(1), 43 (2011).

6. S.F. Muldoon, F. Pasqualetti, S. Gu, M. Cieslak, S.T. Grafton, J.M. Vettel, D.S. Bassett, PLoS Comput. Biol. 12(9), e1005076 (2016).

7. F. Hutchings, C.E. Han, Keller, PLoS Computational Biology 11(12), e1004642 (2015).

8. P. Sanz-Leon, S. A. Knock, A. Spiegler, and V. K Jirsa NeuroImage 111, 385 (2015).

9. S.H. Strogatz, Physica D 143, 1 (2000).

10. V. Vuksanović, P. Hövel, in Selforganization in Complex Systems: The Past, Present, and Future of Synergetics, Proc. of the International Symposium, Hanse Institute of Advanced Studies Delmenhorst, ed. by A. Pelster, G. Wunner (Springer, Berlin, 2016), Understanding Complex Systems, pp. 345-352.

11. V. Vuksanović, P. Hövel, NeuroImage 97, 1 (2014).

12. V. Vuksanović, P. Hövel, Chaos 25, 023116 (2015).

13. J. Cabral, E. Hugues, O. Sporns, G. Deco, NeuroImage 57(1), 130 (2011).

14. J. Cabral, H. Luckhoo, M.W. Woolrich, M. Joensson, H. Mohseni, A. Baker, M. L. Kringelbach, G. Deco, NeuroImage 90, 423 (2014).

15. S.L. Bressler, V. Menon, Trends in Cognitive Sciences 14(6), 277 (2010).

16. M. Breakspear, S. Heitmann, A. Daffertshofer, Front. Hum. Neurosci 4(190), 10 (2010).

17. M. Wildie, M. Shanahan, in Neural Networks (IJCNN), The 2012 International Joint Conference on (IEEE, 2012), pp. 1-6.

18. F. Váša, M. Shanahan, P. J. Hellyer, G. Scott, J. Cabral, R. Leech, NeuroImage 118, 456 (2015).

19. J. Cabral, H. .M. Fernandes, T. J. Van Hartevelt, A. c. James, M. L. Kringelbach, Chaos 23, $046111(2013)$.

20. M. Shanahan, Chaos 20(1), 013108 (2010).

21. N. Kanwisher, Proc. Natl. Acad. Sci. U.S.A. 107(25), 11163 (2010).

22. J.D. Schall, Annual Review of Psychology 55(1), 23 (2004).

23. O. Sporns, Dialogues Clin. Neurosci. 15(3), 247 (2013).

24. J.D. Haynes, G. Rees, Nat. Rev. Neurosci. 7(7), 523 (2006). 
25. H. Farooq, J. Xu, J.W. Nam, D.F. Keefe, E. Yacoub, T. Georgiou, C. Lenglet, Sci. Rep. 6, 38927 (2016).

26. M. Xia, J. Wang, Y. He, PLoS ONE 8(7), 1 (2013). See also http://www.nitrc.org/ projects/bnv/.

27. Y. Iturria-Medina, R.C. Sotero, E.J. Canales-Rodríguez, Y. Alemán-Gómez, L. MelieGarcía, NeuroImage 40(3), 1064 (2008).

28. O. Sporns, G. Tononi, R. Kötter, PLoS Comput. Biol. 1(4), e42 (2005).

29. D.J. Felleman, D.C. Van Essen, Cerebral Cortex 1(1), 1 (1991).

30. O. Ciccarelli, M. Catani, H. Johansen-Berg, C. Clark, A. Thompson, The Lancet Neurology $\mathbf{7}(8), 715$ (2008).

31. J.D. Clayden, Funct. Neurol. Functional Neurology 28(3), 197 (2013).

32. S. Jbabdi, S.N. Sotiropoulos, S.N. Haber, D.C. Van Essen, T.E. Behrens, Nat. Neurosci. $\mathbf{1 8}(11), 1546(2015)$.

33. N. Tzourio-Mazoyer, B. Landeau, D. Papathanassiou, F. Crivello, O. Etard, N. Delcroix, B. Mazoyer, M. Joliot, NeuroImage 15(1), 273 (2002).

34. J. Talairach, P. Tournoux, Co-planar stereotaxic atlas of the human brain, Thieme (1988).

35. D.J. Heeger, D. Ress, Nat. Rev. Neurosci. 3(2), 142 (2002).

36. D.N. Greve, G.G. Brown, B.A. Mueller, G. Glover, T.T. Liu, Psychometrika 78(3), 396 (2013).

37. J.D. Power, A. Mitra, T.O. Laumann, A.Z. Snyder, B.L. Schlaggar, S.E. Petersen, NeuroImage $\mathbf{8 4 ,} 320(2014)$

38. F. Kruggel, D.Y. von Cramon, X. Descombes, NeuroImage 10(5), 530 (1999).

39. A.E. Desjardins, K.A. Kiehl, P.F. Liddle, NeuroImage 13(4), 751 (2001).

40. M. Rubinov, O. Sporns, NeuroImage 52(3), 1059 (2010).

41. E.T. Bullmore, D.S. Bassett, Annual Review of Clinical Psychology 7, 113 (2011).

42. Y. Liu, M. Liang, Y. Zhou, Y. He, Y. Hao, M. Song, C. Yu, H. Liu, Z. Liu, T. Jiang, Brain 131(4), 945 (2008).

43. H. Onias, A. Viol, F. Palhano-Fontes, K.C. Andrade, M. Sturzbecher, G. Viswanathan, D.B. de Araujo, Epilepsy \& Behavior 38, 71 (2014).

44. J. Cabral, M.L. Kringelbach, G. Deco, Prog. Neurobiol. 114, 102 (2014).

45. O. Sporns, Networks of the brain (MIT Press, Cambridge, MA, USA, 2011). 
46. B. Biswal, F.Z. Yetkin, V.M. Haughton, J.S. Hyde, Magn. Reson. Med. 34(4), 537 (1995).

47. M.J. Lowe, Magn. Reson. Mater. Phy. 23(5), 279 (2010).

48. D.M. Cole, S.M. Smith, C.F. Beckmann, Front. Syst. Neurosci. 4, 8 (2010).

49. M.P. van den Heuvel, H. Hulshoff Pol, European Neuropsychopharmacology 20(8), 519 (2010).

50. E. Tagliazucchi, R. Carhart-Harris, R. Leech, D. Nutt, D.R. Chialvo, Hum. Brain Mapp. 35(11), 5442 (2014).

51. R. Carhart-Harris, S. Muthukumaraswamy, L. Roseman, M. Kaelen, W. Droog, K. Murphy, E. Tagliazucchi, E.E. Schenberg, T. Nest, C. Orban, R. Leech, L.T. Williams, T.M. Williams, M. Bolstridge, B. Sessa, J. McGonigle, M.I. Sereno, D. Nichols, P.J. Hellyer, P. Hobden, J. Evans, K.D. Singh, R.G. Wise, H.V. Curran, A. Feilding, D.J. Nutt, Proc. Natl. Acad. Sci. USA 113(17), 4853 (2016).

52. A. Viol, F. Palhano-Fontes, H. Onias, D.B. de Araujo, G. Viswanathan, Sci. Rep. 7, 7388 (2017).

53. J.D. Rudie, J.A. Brown, D. Beck-Pancer, L.M. Hernandez, E.L. Dennis, P.M. Thompson, S.Y. Bookheimer, M. Dapretto, NeuroImage: Clinical 2, 79 (2013).

54. M. Rubinov, S.A. Knock, C.J. Stam, S. Micheloyannis, A.W.F. Harris, L.M. Williams, M. Breakspear, Hum. Brain Mapp. 30(2), 403 (2009).

55. J. Schrouff, V. Perlbarg, M. Boly, G. Marrelec, P. Boveroux, A. Vanhaudenhuyse, M.A. Bruno, S. Laureys, C. Phillips, M. Pélégrini-Issac, P. Maquet, H. Benali, NeuroImage $\mathbf{5 7}(1), 198(2011)$.

56. T.T. Dang-Vu, M. Schabus, M. Desseilles, G. Albouy, M. Boly, A. Darsaud, S. Gais, G. Rauchs, V. Sterpenich, G. Vandewalle, J. Carrier, G. Moonen, E. Balteau, C. Degueldre, A. Luxen, C. Phillips, P. Maquet, Proc. Natl. Acad. Sci. USA 105(39), 15160 (2008).

57. Q. Noirhomme, A. Soddu, R. Lehembre, A. Vanhaudenhuyse, P. Boveroux, M. Boly, S. Laureys, Front. Syst. Neurosci. 4, 160 (2010).

58. Z. Huang, R. Dai, X. Wu, Z. Yang, D. Liu, J. Hu, L. Gao, W. Tang, Y. Mao, Y. Jin, X. Wu, B. Liu, Y. Zhang, L. Lu, S. Laureys, X. Weng, G. Northoff, Hum. Brain Mapp. 35(5), 1997 (2014).

59. P. Barttfeld, L. Uhrig, J.D. Sitt, M. Sigman, B. Jarraya, S. Dehaene, Proc. Natl. Acad. Sci. USA 112(3), 887 (2015). 
60. G. Deco, V.K. Jirsa, A.R. McIntosh, Trends in Neurosciences 36(5), 268 (2013).

61. M.A. Koch, D.G. Norris, M. Hund-Georgiadis, NeuroImage 16(1), 241 (2002).

62. A. Pikovsky, M.G. Rosenblum, J. Kurths, Synchronization, A Universal Concept in Nonlinear Sciences (Cambridge University Press, Cambridge, 2001).

63. S. Boccaletti, J. Kurths, G. Osipov, D.L. Valladares, C.S. Zhou, Phys. Rep. 366, 1 (2002).

64. E. Mosekilde, Y. Maistrenko, D. Postnov, Chaotic Synchronization: Applications to Living Systems (World Scientific, Singapore, 2002).

65. A.G. Balanov, N.B. Janson, D.E. Postnov, O.V. Sosnovtseva, Synchronization: From Simple to Complex (Springer, Berlin, 2009).

66. E. Rossoni, Y. Chen, M. Ding, J. Feng, Phys. Rev. E 71(6), 061904 (2005).

67. Q.Y. Wang, Q.S. Lu, Chin. Phys. Lett. 22(3), 543 (2005).

68. C. Hauptmann, O.E. Omel'chenko, O. Popovych, Y. Maistrenko, P. Tass, Phys. Rev. E 76, 066209 (2007).

69. C. Masoller, M.C. Torrent, J. García-Ojalvo, Phys. Rev. E 78, 041907 (2008).

70. Q. Wang, Q. Lu, G. Chen, Int. J. Bifur. Chaos 18(4), 1189 (2008).

71. Q. Wang, Q. Lu, G. Chen, Z. Feng, L. Duan, Chaos, Solitons and Fractals 39(2), 918 (2009).

72. C. Masoller, M.C. Torrent, J. García-Ojalvo, Philosophical Transactions of the Royal Society A: Mathematical, Physical and Engineering Sciences 367(1901), 3255 (2009).

73. D.V. Senthilkumar, J. Kurths, M. Lakshmanan, Chaos 19(2), 023107 (2009).

74. X. Liang, M. Tang, M. Dhamala, Z. Liu, Phys. Rev. E 80, 066202 (2009).

75. J. Lehnert, T. Dahms, P. Hövel, E. Schöll, Europhys. Lett. 96, 60013 (2011).

76. O. Popovych, S. Yanchuk, P. Tass, Phys. Rev. Lett. 107, 228102 (2011).

77. A.L. Hodgkin, A.F. Huxley, J. Physiol. 117, 500 (1952).

78. R. FitzHugh, Biophys. J. 1, 445 (1961).

79. J. Nagumo, S. Arimoto, S. Yoshizawa., Proc. IRE 50, 2061 (1962).

80. A. Bergner, M. Frasca, G. Sciuto, A. Buscarino, E. J. Ngamga, L. Fortuna, J. Kurths, Phys. Rev. E 85, 026208 (2012).

81. M. Breakspear, J. A. Roberts, J. .R. Terry, S. Rodrigues, N. Mahant, P. A. Robinson, Cerebral Cortex 16(9), 1296 (2006). 
82. V. Nicosia, M. Valencia, M. Chavez, A. Díaz-Guilera, V. Latora, Phys. Rev. Lett. 110, $174102(2013)$.

83. A. Arenas, A. Díaz-Guilera, C.J. Pérez Vicente, Phys. Rev. Lett. 96, 114102 (2006).

84. V.K. Jirsa, H. Haken, Phys. Rev. Lett. $77(5), 960$ (1996).

85. E.M. Izhikevich, IEEE Transactions on Neural Networks 15(5), 1063 (2004).

86. Y. Kuramoto, in International symposium on mathematical problems in theoretical physics, Lecture Notes in Physics, vol. 39, ed. by H. Araki (Springer, 1975), Lecture Notes in Physics, vol. 39, pp. 420-422.

87. J.A. Acebrón, L.L. Bonilla, C.J. Pérez Vicente, F. Ritort, R. Spigler, Rev. Mod. Phys. 77, 137 (2005).

88. F.A. Rodrigues, T.K.D. Peron, P. Ji, J. Kurths, Phys. Rep. 610, 1 (2016).

89. P.J. Hellyer, M. Shanahan, G. Scott, R.J.S. Wise, D.J. Sharp, R. Leech, J. Neurosci. 34(2), 451 (2014).

90. G. Deco, M. L. Kringelbach, Neuron 84, 892 (2014).

91. J. Cabral, E. Hugues, M.L. Kringelbach, G. Deco, NeuroImage 62, 1342 (2012).

92. M. Breakspear, S. Heitmann, A. Daffertshofer, Front. Hum. Neurosci. 4(190), 1 (2010).

93. A. Keane, T. Dahms, J. Lehnert, S.A. Suryanarayana, P. Hövel, E. Schöll, Eur. Phys. J. B $\mathbf{8 5}(12), 407$ (2012).

94. V. Vuksanović, P. Hövel, Cogn. Neurodyn. 10(4), 361 (2016).

95. G. Deco, V.K. Jirsa, J. Neurosci. 32(10), 3366 (2012).

96. J.S. Damoiseaux, S.A.R.B. Rombouts, F. Barkhof, P. Scheltens, C.J. Stam, S.M. Smith, C.F. Beckmann, Proc. Natl. Acad. Sci. U.S.A. 103(37), 13848 (2006).

97. K. Friston, A. Mechelli, R. Turner, C.J. Price, NeuroImage 12(4), 466 (2000).

98. A.K. Seth, P. Chorley, L.C. Barnett, NeuroImage 65, 540 (2013).

99. K. Friston, R.J. Dolan, NeuroImage 52(3), 752 (2010).

100. E. Tognoli, J.S. Kelso, Neuron 81(1), 35 (2014).

101. E.T. Bullmore, O. Sporns, Nat. Rev. Neurosci. 10(3), 186 (2009).

102. B.B. Biswal, et al., Proc. Natl. Acad. Sci. USA 107(10), 4734 (2010).

103. M. Demirtas, G. Deco in Computational Psychiatry ed. by A. Anticevic, J. .D. Murray (Academic Press, London, 2018), pp. 87-116. 
${ }_{685}$ A List of cortical and sub-cortical regions

Table 1 Cortical and sub-cortical regions according to the automated anatomic labelling (AAL) template image [33]. Indexes from 1-45 and 46-90 indicate right (R) and left (L) hemisphere respectively, and refer to the order in which the brain regions of interest are arranged in all connectivity, adjacency and distance matrices of this paper.

\begin{tabular}{|c|c|c|}
\hline Index $\mathrm{R} / \mathrm{L}$ & Anatomical Description & Label \\
\hline $1 / 46$ & Precentral & PRE \\
\hline $2 / 47$ & Frontal Sup & F1 \\
\hline $3 / 48$ & Frontal Sup Orb & F10 \\
\hline $4 / 49$ & Frontal Mid & F2 \\
\hline $5 / 50$ & Frontal Mid Orb & F20 \\
\hline $6 / 51$ & Frontal Inf Oper & F30P \\
\hline $7 / 52$ & Frontal Inf Tri & F3T \\
\hline $8 / 53$ & Frontal Inf Orb & F30 \\
\hline $9 / 54$ & Rolandic Oper & $\mathrm{RO}$ \\
\hline $10 / 55$ & Supp Motor Area & SMA \\
\hline $11 / 56$ & Olflactory & $\mathrm{OC}$ \\
\hline $12 / 57$ & Frontal Sup Medial & F1M \\
\hline $13 / 58$ & Frontal Mid Orb & SMG \\
\hline $14 / 59$ & Gyrus Rectus & GR \\
\hline $15 / 60$ & Insula & IN \\
\hline $16 / 61$ & Cingulum Ant & ACIN \\
\hline $17 / 62$ & Cingulum Mid & MCIN \\
\hline $18 / 63$ & Cingulum Post & PCIN \\
\hline $19 / 64$ & Hippocampus & HIP \\
\hline $20 / 65$ & ParaHippocampal & PHIP \\
\hline $21 / 66$ & Amygdala & AMYG \\
\hline $22 / 67$ & Calcarine & V1 \\
\hline $23 / 68$ & Cuneus & Q \\
\hline $24 / 69$ & Lingual & LING \\
\hline $25 / 70$ & Occipital Sup & O1 \\
\hline $26 / 71$ & Occipital Mid & $\mathrm{O} 2$ \\
\hline $27 / 72$ & Occipital Inf & O3 \\
\hline $28 / 73$ & Fusiform & FUSI \\
\hline $29 / 74$ & Postcentral & POST \\
\hline $30 / 75$ & Parietal Sup & P1 \\
\hline $31 / 76$ & Parietal Inf & P2 \\
\hline $32 / 77$ & Supra Marginal Gyrus & SMG \\
\hline $33 / 78$ & Angular & $\mathrm{AG}$ \\
\hline $34 / 79$ & Precuneus & $\mathrm{PQ}$ \\
\hline $35 / 80$ & Paracentral Lobule & PCL \\
\hline $36 / 81$ & Caudate & CAM \\
\hline $37 / 82$ & Putamen & PUT \\
\hline $38 / 83$ & Pallidum & PAL \\
\hline $39 / 84$ & Thalamus & THA \\
\hline $40 / 85$ & Heschi & HES \\
\hline $41 / 86$ & Temporal Sup & $\mathrm{T} 1$ \\
\hline $42 / 87$ & Temporal Pole sup & $\mathrm{T} 1 \mathrm{P}$ \\
\hline $43 / 88$ & Temporal Mid & $\mathrm{T} 2$ \\
\hline $44 / 89$ & Temporal Pole Mid & $\mathrm{T} 2 \mathrm{P}$ \\
\hline $45 / 90$ & Temporal Inf & T3 \\
\hline
\end{tabular}

